\title{
Risk of psychopathology in adolescent offspring of mothers with psychopathology and recurrent depression ${ }^{\dagger}$
}

Ruth Sellers, Stephan Collishaw, Frances Rice, Ajay K. Thapar, Robert Potter, Becky Mars, Gordon T. Harold, Daniel J. Smith, Michael J. Owen, Nick Craddock and Anita Thapar

\section{Background}

Offspring of mothers with depression are at heightened risk of psychiatric disorder. Many mothers with depression have comorbid psychopathology. How these co-occurring problems affect child outcomes has rarely been considered.

\section{Aims \\ To consider whether the overall burden of co-occurring psychopathology in mothers with recurrent depression predicts new-onset psychopathology in offspring.}

\section{Method}

Mothers with recurrent depression and their adolescent offspring (9-17 years at baseline) were assessed in 2007 and on two further occasions up to 2011. Mothers completed questionnaires assessing depression severity, anxiety, alcohol problems and antisocial behaviour. Psychiatric disorder in offspring was assessed using the Child and Adolescent Psychiatric Assessment

\section{Results}

The number of co-occurring problems in mothers $(0,1$ or $2+)$ predicted new-onset offspring disorder (odds ratio $(\mathrm{OR})=1.80,95 \% \mathrm{Cl} 1.17-2.77, P=0.007$ ). Rates varied from 15.7 to $34.8 \%$ depending on the number of co-occurring clinical problems. This remained significant after controlling for maternal depression severity $(\mathrm{OR}=1.73$, $95 \% \mathrm{Cl} 1.03-2.89, P=0.040)$.

\section{Conclusions}

The burden of co-occurring psychopathology among mothers with recurrent depression indexes increased risk of future onset of psychiatric disorder for offspring. This knowledge can be used in targeting preventive measures in children at high risk of psychiatric disorder

\section{Declaration of interest}

D.J.S. has received honoraria for speaking at educational meetings organised by Astrazeneca and Lilly.
Children of mothers with depression are at an increased risk of developing psychopathology, with a two- to fivefold increase in risk compared with offspring of mothers without depression. ${ }^{1,2}$ However, there is considerable heterogeneity in outcome and it is important to gain a better understanding about which aspects of maternal illness are associated with an elevated risk for offspring, ${ }^{3}$ as this will help better target preventive interventions to those at greatest risk of developing psychopathology. Features of depressive illness in mothers (such as severity and chronicity) explain some of the variation in risk for psychopathology in offspring. ${ }^{4-7}$ However, depression is often accompanied by additional psychopathology, ${ }^{8}$ of which the most common are anxiety, ${ }^{9}$ antisocial behaviour ${ }^{10}$ and substance misuse. ${ }^{11,12}$ These comorbid problems have each been associated with increased impairment in the affected adult, and with more severe depression symptoms compared with depression alone. ${ }^{13-15}$ An understanding of the implications of these co-occurring problems in mothers for psychopathology in their offspring is limited. Importantly, it is not yet known whether co-occurring psychopathology in mothers with depression indexes risk of child psychopathology in addition to severity of maternal depression. If co-occurring psychopathology in adults with depression is a separate index for a more severe familial depression phenotype, this would be important in guiding intervention strategies for high-risk children. Recent research by McLaughlin et al ${ }^{16}$ utilised data from the World Mental Health Surveys, selected to be nationally or regionally representative, and demonstrated that total number of maternal disorders (depression, panic disorder, generalised anxiety disorder, substance dependence and antisocial personality disorder) increased risk for a psychiatric disorder in offspring. A

'See editorial, pp. 84-85, this issue. complementary approach is to assess psychopathology in a high-risk group of mothers with recurrent depression and their offspring.

We utilised data from a longitudinal study of children of mothers with recurrent depression. Longitudinal research is scarce, but particularly informative; it is possible to assess the impact of maternal depression and additional problems on offspring psychopathology as it develops. Thus, risk markers for the onset of psychopathology in offspring can be identified. The primary aim of the study was to investigate whether the number of co-occurring clinical problems (anxiety, antisocial behaviour and harmful drinking) in mothers with recurrent depression indexed increased risk of new-onset psychopathology in offspring, and whether any increased risk found was independent of variation in maternal depression severity. We hypothesised that comorbid problems in mothers with recurrent depression would increase the risk of psychopathology in offspring over and above any association with maternal depression severity.

\section{Method}

\section{Participants}

Analyses utilised data from the three-wave Early Prediction of Adolescent Depression (EPAD) study, an observational community study of the offspring of parents with recurrent depression. Parents were recruited predominantly from primary care in South Wales, UK, on the basis of treatment for at least two episodes of depression (78\%); from a previous community study of recurrent unipolar depression (19\%); and from additional sources such as posters within primary care settings $(3 \%)$. Participants did not differ on predictor or outcome variables according to the sampling method. 
A history of recurrent depression in the parent (two or more episodes of DSM-IV ${ }^{17}$ major depression) was confirmed at interview. Families were excluded if the parent had a diagnosis of bipolar disorder during screening or at interview, the parent with clinical depression was not biologically related to the child, the child did not live at home at baseline, or the child had moderate to severe intellectual disability (IQ $<50)$. To provide coverage over the whole of adolescence across the course of the study, families were selected if children were aged between 9 and 17 years at the first assessment. Where there was more than one eligible child per household, the youngest child was selected given the focus on new-onset child psychopathology over adolescence, and to avoid bias in parental selection of children.

The EPAD study includes 337 parents with recurrent depression (315 mothers, 22 fathers; age 26-55 years, mean 41.7 years) and their children (197 females, 140 males; age 9-17 years, mean 12.4 years). Inclusion criteria and retention to follow-up for the present analyses are summarised in Fig. 1. Given the small number of fathers with depression participating in the study, analyses were conducted with mothers only $(n=315)$. Preliminary analyses identified 73 children $(23 \%)$ with a disorder at baseline (Table 1). This represents a rate in excess of that found in the general population $(11.5 \%$ for ages $11-16) .{ }^{18}$ The focus in the present paper was on the prediction of 'new-onset' offspring disorders over the course of the study. Analyses were therefore restricted to the subsample of children without a psychiatric disorder at baseline $(n=242 / 315)$.
Seventeen further families were excluded due to incomplete data on baseline mother psychopathology and two families were excluded where the affected parent was diagnosed with bipolar disorder by follow-up. Of the eligible baseline sample $(n=223)$, $219(98.2 \%)$ families participated in the first follow-up assessment approximately 16 months later (s.d. $=2.69)$. A second follow-up was conducted a further 12 months later (s.d.=1.56); here 214/ $223(96.0 \%)$ families participated. Only two families declined to participate at both follow-up assessments, with a further 12 families having incomplete data. Therefore complete data was available on child outcomes for 209/223 (93.7\%) families.

Families with missing data at either of the follow-ups $(n=14)$ were compared on key variables with those with complete data $(n=209)$. There were no differences in terms of parent and child age and gender, parental depression age at onset and past impairment, or number of co-occurring problems. Missing data at follow-up were however associated with higher baseline maternal depression severity (Beck Depression Inventory, BDI ${ }^{19}$ and with longer duration of previous depressive episodes (online Table DS1).

\section{Procedure}

The majority of assessments were conducted in the family home (99.6\%), with parents and children assessed by different interviewers. Parents and children were given a description of the study and provided written informed consent or written assent (if under 16 years of age). Assessments lasted approximately $3 \mathrm{~h}$

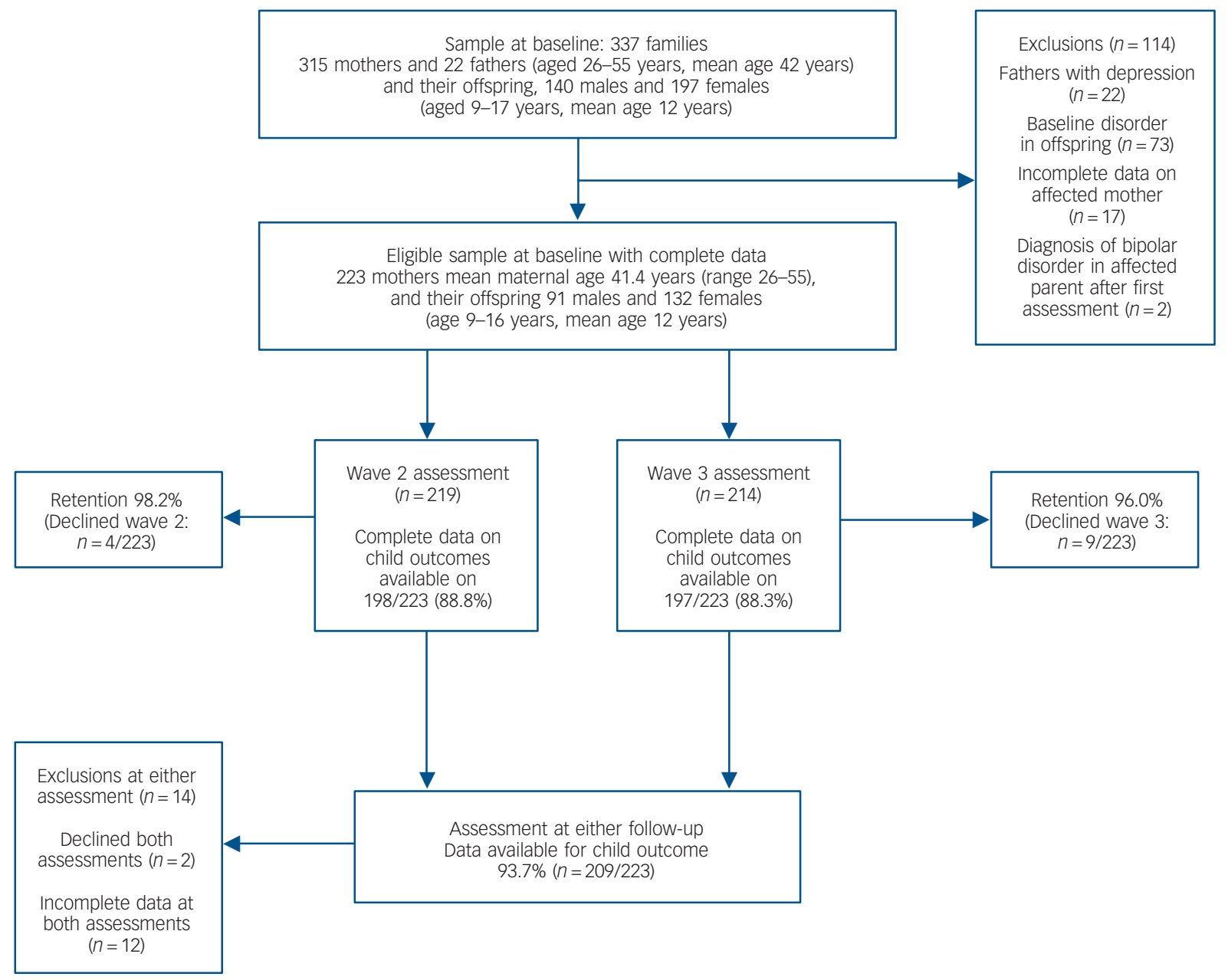

Fig. 1 Flow chart showing retention at each assessment in the current study. 
Table 1 Baseline rates of disorder in offspring of mothers with depression, and new onset of any psychiatric disorder at each follow-up

\begin{tabular}{|lccc|} 
& & $n(\%)$ & \\
\cline { 2 - 4 } Disorder & Baseline $(n=315)$ & New-onset wave 2 $(n=198)$ & New-onset wave $3(n=197)$ \\
\hline Any disorder & $73(23.2)$ & $33(16.7)$ & $11(5.6)$ \\
\hline Depressive disorder & $15(4.8)$ & $11(5.6)$ & $3(1.5)$ \\
\hline Anxiety disorder & $34(10.8)$ & $20(10.1)$ & $8(4.1)$ \\
\hline Disruptive behaviour disorder & $22(7.0)$ & $12(6.1)$ & $6(3.0)$ \\
\hline Bipolar spectrum disorder & $1(0.3)$ & 0 & 0 \\
\hline Attention-deficit hyperactivity disorder & $20(6.3)$ & 0 & 0 \\
\hline Eating disorder & $3(1.0)$ & 0 & 0 \\
\hline
\end{tabular}

and participants were compensated for their time. Interviewers were aware that all parents had a history of recurrent depression but were masked to any additional clinical problems in parent and child psychopathology. Interviewers were trained psychology graduates supervised weekly by psychiatrists. Ethical approval was provided by the multi-regional ethics committee.

\section{Measures}

Maternal depression

A semi-structured interview, the Schedules for Clinical Assessment in Neuropsychiatry, ${ }^{20}$ was used to establish the presence of DSMIV major depression over the preceding month. In total 21\% (47/ 223) of mothers met criteria for a major depressive episode at baseline. Mothers completed the $\mathrm{BDI}^{19}$ to assess severity of symptoms of baseline depression. The BDI is a 21 -item questionnaire with each item scored $0-3$ according to severity of symptoms that day, with a maximum score of 63 . A total depression severity score was calculated (range $0-49$, mean 15.48, s.d. $=10.64$, $\alpha=0.91$.

At the baseline assessment mothers also provided information on the clinical features of their past depressive episodes. A detailed description is provided by Mars et al. ${ }^{7}$ Briefly, mothers provided information about age at first episode of depression, number of episodes, and duration of each episode. They also rated impairment during worst and second-worst depressive episodes using the Global Assessment of Functioning scale ${ }^{20}$ according to functioning in psychosocial, occupational and relationships domains, with scores ranging from 0 to 100 (lower scores indicating greater impairment).

\section{Maternal anxiety}

Maternal anxiety was assessed using the Hospital Anxiety and Depression Scale (HADS) anxiety subscale. ${ }^{21}$ Seven items were scored from 0 to 3 according to severity of anxiety symptoms over the preceding week. A total anxiety symptom score was calculated $(\alpha=0.82)$. A dichotomous variable was created using the previously validated cut-off score of 11 or more, indicative of clinically significant anxiety. ${ }^{21}$

\section{Maternal antisocial behaviour}

Mothers reported on their own current antisocial behaviour using 33 items of the 34-item Adult Self Report (ASR) questionnaire, ${ }^{22}$ with items coded 0 (absent), 1 (somewhat/sometimes true) or 2 (very true). The item 'I have never been arrested' was excluded as this item referred to lifetime behaviour. A total antisocial behaviour score was calculated (observed range $0-23, \alpha=0.75$ ). A dichotomous variable was created using a cut-off of 1 standard deviation above the sample mean $(\geqslant 13)$ as indicative of significant antisocial behaviour symptoms as there is no established cut-off point for this scale.

\section{Maternal alcohol use}

Alcohol use was measured using the widely used and wellvalidated Alcohol Use Disorders Identification Test (AUDIT), ${ }^{23}$ a 10 -item questionnaire assessing harmful drinking within the past year. Each item is scored $0-4$ and a total AUDIT score was calculated (observed range $0-25, \alpha=0.78$ ). Harmful drinking was coded according to established criteria for women of a score of 13 or greater. $^{23}$

\section{Number of maternal co-occurring clinical problems at baseline}

An index of 'co-occurring problems' was created to tap the breadth of co-occurring psychopathology currently experienced by mothers with recurrent depression. The number of problems (anxiety, antisocial behaviour and harmful drinking) that reached the thresholds outlined above were summed, leading to a maximum of three possible concurrent problems in addition to recurrent depression. At baseline 59.6\% mothers $(n=133 / 223)$ reported no concurrent problem, 28.7\% $(n=64)$ mothers reported one concurrent problem, $10.8 \%(n=24)$ reported two concurrent problems and $0.9 \%$ mothers $(n=2)$ reported three concurrent problems. Analyses compared those with recurrent depression only, one additional clinical problem or multiple additional problems (two or three additional clinical problems; $11.7 \%, n=26)$.

\section{Child psychopathology at follow-up}

Child psychiatric disorder was assessed using parent and child versions of the Child and Adolescent Psychiatric Assessment $(\mathrm{CAPA}),{ }^{24}$ a semi-structured research diagnostic interview providing a detailed assessment of child psychopathology over the preceding 3 months. The study considered DSM-IV diagnoses of depressive disorders (major depressive disorder, dysthymia, depression not otherwise specified (NOS) and adjustment disorder), anxiety disorders (generalised anxiety disorder, separation anxiety, social phobia, panic disorder, agoraphobia and obsessive-compulsive disorder), disruptive behaviour disorders (oppositional defiant disorder, conduct disorder and disruptive behaviour NOS) and attention-deficit hyperactivity disorder (ADHD). Eating disorders, cyclothymia and bipolar disorder were also assessed. Interrater reliabilities for child disorder were excellent (average $\kappa=0.92$ ). Case notes on individuals reaching diagnostic criteria and subthreshold cases were reviewed by two consultant child psychiatrists and DSM-IV diagnoses were agreed by consensus. A disorder was considered to be present if a diagnosis was made by either the mother or child interview. ${ }^{25}$ New-onset cases were defined where children received a DSM-IV psychiatric diagnosis at either of the two follow-ups, but not at baseline. 


\section{Household demographics}

Family poverty and maternal education were assessed by parental self-report questionnaire, and family type (single or two-parent) was assessed at interview. Mothers rated their family's financial situation on a five-point scale from 'living comfortably' to 'finding it very difficult to make ends meet'. This was dichotomised to identify families 'finding it difficult' or 'finding it very difficult' to make ends meet.

\section{Analysis}

Preliminary linear regression analyses assessed whether maternal depression severity (BDI score) was associated with the number of maternal comorbid clinical problems at baseline.

\section{Primary aim}

Binary logistic regression analyses tested the prospective association between number of co-occurring problems and new-onset child disorder at follow-up. Analyses were repeated controlling for mother depression severity (BDI) at baseline. Results are described as odds ratios (ORs) with 95\% confidence intervals. Odds ratios represent the average increase in risk across each step of the three-level predictor $(0,1,2+$ co-occurring problems).

\section{Sensitivity analyses}

Additional sensitivity analyses examined the robustness of findings.

(a) Social adversity may be an important confounding factor. Regression analyses considered whether family financial difficulty was associated with maternal depression severity, the number of co-occurring psychopathology and child new-onset disorder.

(b) We tested whether past course and severity of parents' depressive illness might act as additional confounders of the relationship between co-occurring parent psychopathology and child new-onset disorder over and above any effect of baseline parent depression severity. Analyses assessed associations between several maternal depression features (age at onset, average episode duration, average impairment) and (i) the number of maternal co-occurring problems at baseline $(0,1,2+)$, and (ii) new-onset child disorder, in each case controlling for baseline maternal depression severity.

(c) The CAPA uses a 3-month time frame and therefore some episodes of illness may have been missed. This may be a particular concern in relation to depression given its episodic course. For example, some children may have experienced an episode of depression at some point between assessments but not in the 3 months prior to interview. We may also have incorrectly classified some children as having a new-onset disorder if we had missed episodes of depression prior to baseline. The retrospective DSM-IV depression symptom checklists carried out at each interview allowed us to address these possibilities. Two children who we had not classified as having new-onset disorder reported significant depression symptoms (5+) after baseline but not at interview at either follow-up. Conversely, five children who had been classified as having a new-onset disorder had in fact reported significant symptoms of depression (5+) prior to baseline. Analyses were repeated (i) excluding the five children with possible depression prior to baseline and (ii) including the two additional offspring with possible new-onset depression. As noted later, results were unchanged.
All analyses included child (baseline) age and gender as covariates. Analyses were conducted using Stata version 10 for Windows using multiple imputation (multivariate imputation by chained equations (MICE) procedure with 10 imputed datasets ${ }^{26}$ ) to account for incomplete data at follow-up for a small number of individuals. For clarity and ease of interpretation, non-imputed numbers and percentages are presented. A $P \leqslant 0.05$ was used as the established significance level.

\section{Results}

\section{Co-occurring psychopathology in mothers with recurrent depression}

Table 2 shows characteristics of psychopathology in mothers at baseline and sample demographics. In total $40 \%$ of this sample of mothers with recurrent depressive disorder also had significant other problems - anxiety, antisocial behaviour and/or harmful drinking.

The number of co-occurring clinical problems in the mothers was associated with maternal depression severity as measured by the BDI at baseline $(\beta=0.86, b=9.38,95 \%$ CI $7.82-10.97$, $P \leqslant 0.001)$. On average, each additional co-occurring clinical problem was associated with a 9.4-point increase in BDI score at baseline.

\section{New-onset offspring psychiatric disorder}

Among children free of disorder at baseline, 21.1\% (44/209) experienced new-onset disorders across the follow-up period. These included 14 depressive disorders, 28 anxiety disorders (excluding specific phobia), 18 disruptive behaviour disorders and 3 adjustment disorders. Rates of new-onset disorder by age and gender are summarised in Table 3.

\section{Does the number of co-occurring problems in mothers predict new-onset disorder in offspring?}

Figure 2 shows that at follow-up, rates of new-onset disorder in offspring increased according to the number of additional clinical

\section{Table 2 Parent demographics and clinical profile at baseline assessment ${ }^{a}$}

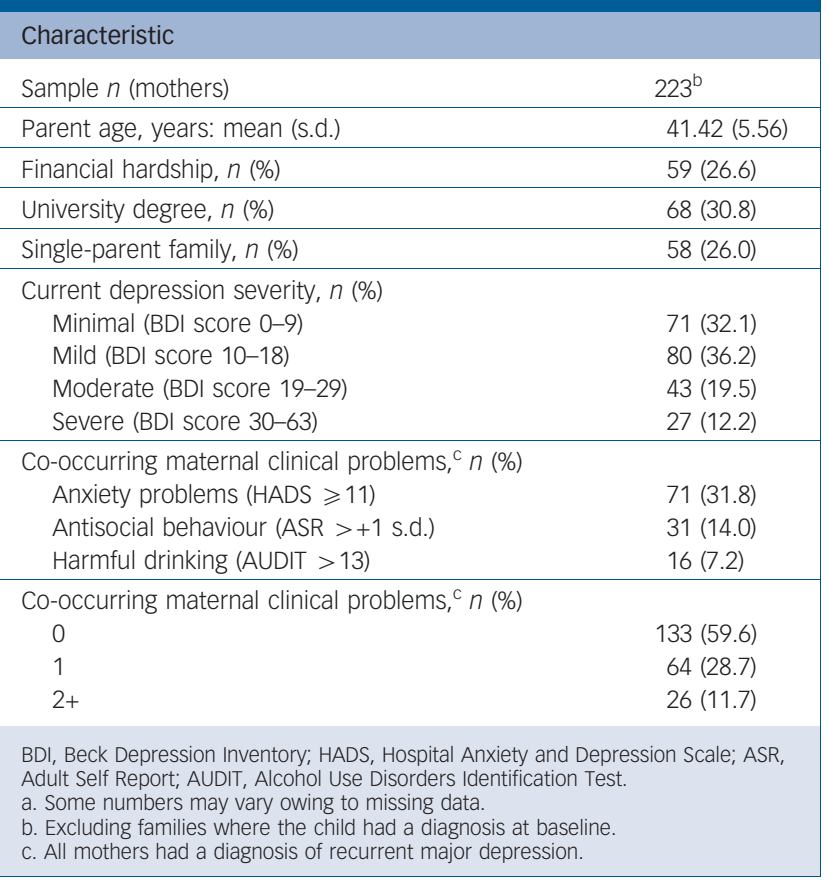




\begin{tabular}{|c|c|c|c|c|c|c|}
\hline \multirow[b]{2}{*}{ Offspring age at baseline, years } & \multicolumn{2}{|c|}{ Total } & \multicolumn{2}{|c|}{ Females } & \multicolumn{2}{|c|}{ Males } \\
\hline & $n / N$ & $\%$ & $n / N$ & $\%$ & $n / N$ & $\%$ \\
\hline $9-11$ & $11 / 80$ & 13.8 & $8 / 49$ & 16.3 & $3 / 31$ & 9.7 \\
\hline $12-14$ & $26 / 95$ & 27.4 & $16 / 53$ & 30.2 & $10 / 42$ & 23.8 \\
\hline $15-16$ & 7/34 & 20.6 & $4 / 21$ & 19.0 & $3 / 13$ & 23.1 \\
\hline Total & $44 / 209$ & 21.1 & $28 / 123$ & 22.8 & $16 / 86$ & 18.6 \\
\hline
\end{tabular}

problems in mothers and varied from 15.7 to $34.8 \%$ (no clinical problems $v .2+$ clinical problems).

The number of additional clinical problems in the mother $(0$, $1,2+)$ was associated with new-onset offspring disorder ( $\mathrm{OR}=1.80,95 \%$ CI $1.17-2.77, \quad P=0.007)$. This association remained after adjusting for baseline maternal depression severity ( $\mathrm{OR}=1.73,95 \%$ CI $1.03-2.89, P=0.040)$. The majority of newonset cases of disorder $(54.5 \%, 24 / 44)$ occurred in children whose mothers reported at least one comorbid problem (8/14 depressive disorder; 14/28 anxiety disorder, 12/18 disruptive behaviour disorder). Small sample sizes precluded separate testing for association between mothers' additional clinical problems and each child diagnostic group.

\section{Sensitivity analyses}

(a) Over a quarter of mothers described themselves as having financial difficulties. Those in financial difficulties reported higher depression severity $(\beta=0.28, b=6.64,95 \%$ CI $3.56-$ 9.72, $P \leqslant 0.001)$ and increased co-occurring psychopathology ( $\beta=0.15, b=0.24,95 \%$ CI $0.03-0.44, P=0.026)$. However, financial hardship did not significantly predict new-onset disorder in offspring after adjusting for baseline severity ( $\mathrm{OR}=0.94,95 \%$ CI $0.43-2.07, P=0.875)$.

(b) We tested severity and course of parents' depressive illness prior to baseline as additional potential confounders. Number of co-occurring problems $(0,1,2+)$ in mothers with recurrent depression was associated with a younger age at depression onset (but not longer episode duration or greater impairment) when accounting for baseline maternal depression severity (results available from the authors on request). However, maternal age at onset of depressive disorder did not significantly predict new-onset disorder in offspring ( $\mathrm{OR}=0.96,95 \%$ CI $0.92-1.00, P=0.071$, adjusting for baseline severity).

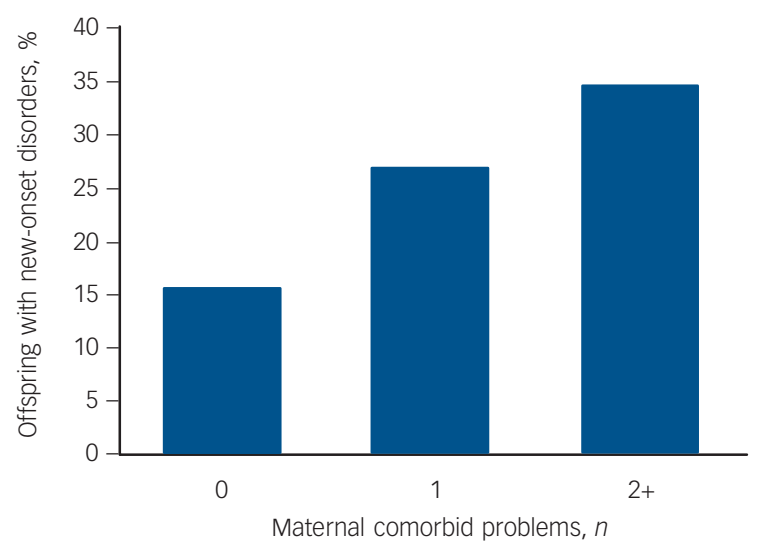

Fig. 2 Rates of new-onset child psychiatric disorder across follow-up by maternal co-occurring psychopathology. (c) Five children reported possible depression prior to the baseline assessment (five or more symptoms of depression). After excluding these individuals from analyses primary findings were unchanged; parent co-occurring problems remained significantly associated with future disorder in offspring after adjusting for maternal depression severity ( $\mathrm{OR}=2.37,95 \%$ CI 1.23-4.57, $P=0.010$ ). Two additional children reported possible depression between baseline and follow-up assessments. Including these two children as having new-onset disorder also made no difference to the findings $(\mathrm{OR}=1.87,95 \% \mathrm{CI} 1.05-3.32, P=0.033)$.

\section{Discussion}

It is well-established that offspring of mothers with depression are at elevated risk of developing depression and other psychiatric disorders. It is less clear how far risk for offspring psychopathology reflects the presence of co-occurring maternal psychiatric problems such as anxiety, antisocial behaviour or problem drinking. The current study utilised a longitudinal high-risk sample of offspring of mothers with recurrent depression and considered prospective associations between additional parent clinical problems (anxiety, antisocial behaviour and harmful drinking) and new-onset offspring psychiatric disorder.

Co-occurring psychopathology in mothers was common and was associated with increased severity of adults' own depression symptoms, consistent with previous research. ${ }^{1315,27}$ Co-occurring psychopathology in mothers was a strong predictor of future disorders in offspring; in this sample more than half of new-onset disorders in offspring occurred for the subgroup of mothers with depression with other clinical problems. This association remained even when mothers' baseline depression severity was controlled for.

Age at onset, chronicity and past severity of parent depression have been found to be associated with child outcomes in this sample $^{7}$ and previous studies. ${ }^{6}$ We therefore considered whether these other characteristics of maternal depression accounted for the increased risk for offspring psychopathology among mothers with co-occurring psychopathology. Age at onset was associated with maternal co-occurring psychopathology, but age at onset did not account for the association between maternal co-occurring psychopathology and the increased risk for offspring new-onset psychopathology. Similarly, differences in reported financial hardship also failed to account for this association.

One possible mechanism is that depression occurring with additional clinical problems indexes higher genetic loading. There is evidence of a shared genetic liability between depression and anxiety, and to a lesser extent depression and alcohol misuse, and depression and antisocial behaviour. ${ }^{28-30}$ Additional clinical problems in the mother may also have detrimental environmentally mediated effects on children's mental health, for example, through impaired parenting. Previous research has shown that parent depression is associated with impairments in parenting that may, in part, mediate the effect of maternal depression on child 
outcome, ${ }^{31}$ although findings are not entirely consistent with other studies suggesting that this link might represent passive gene-environment correlation. ${ }^{32}$ Additional clinical problems in parents with depression are associated with increased impairment, ${ }^{13-15}$ and this increased impairment may well manifest itself in different ways that have a negative impact on the offspring. There is some evidence that recurrent maternal depression accompanied by other psychopathology is associated with less optimal parenting compared with depression alone, at least in mothers of infants. ${ }^{33}$ Antisocial behaviour with depression in mothers has also been found to be associated with less warmth and more hostility compared with mothers with depression alone. ${ }^{34}$ How other clinical problems affect parenting is not clearly understood. Future research should consider the mechanisms that may account for the association between comorbid clinical problems in parents with recurrent depression and child psychopathology as this has important implications for prevention and intervention. Intervention studies that target co-occurring mental health problems in parents as well as the treatment of depression would help to identify whether this results in benefits to offspring as well as parents.

\section{Strengths}

A major strength of the current study is the use of longitudinal data with excellent retention to assess the development of psychopathology in high-risk offspring over time, and thus identify risk markers that are associated with the onset of psychopathology in adolescent offspring. To our knowledge, no previous study has assessed the cumulative burden of clinical problems in mothers with recurrent depression, and the impact on child disorder over time.

\section{Limitations}

This study should be considered in light of several limitations.

First, the CAPA assesses offspring psychopathology in the preceding 3 months. We were unable to comprehensively assess lifetime diagnoses for the offspring and thus any diagnoses prior to the baseline assessment, or between baseline and follow-up that subsequently remitted, may have been missed. This may be a particular concern in relation to depression given its episodic course. Additional sensitivity tests did show that findings were unchanged when we excluded a small number of children who at baseline reported high levels of prior depression symptoms or when we included two additional children as having new-onset disorder based on retrospective reports of symptoms at follow-up.

Second, a wide age range of children were included in the study. Not all had passed fully through the period of risk for onset of depression and other disorders, and may go on to develop problems in the future. The study may thus represent an underestimate of the intergenerational risk for psychopathology in this population.

Third, only two follow-up assessments were conducted and this combined with variation in follow-up intervals meant it was not feasible to undertake more sophisticated methods such as survival analysis.

Fourth, given the already significant burden of interview assessments, it was only possible to assess maternal anxiety, antisocial behaviour and alcohol misuse using questionnaires rather than diagnostic interviews. Again, it seems likely that findings are somewhat conservative, as co-occurring disorders meeting stringent diagnostic criteria would be expected to be even more strongly associated with offspring disorder. However, self-report measures of mental health are commonly used in primary care and thus the findings of this study are relevant to the contexts where most adults with depression are assessed and treated. The current study did not assess maternal drug misuse, another common co-occurring problem in adults with depression. $^{12}$

Fifth, co-occurring psychopathology in mothers was independently associated with concurrent depression severity (as measured by the BDI) and an earlier age at depression onset. Analyses suggest that these factors do not account for the observed differences in risk for offspring. However, despite these controls it remains possible that the study has not fully accounted for residual variation in parental depression illness course, severity and impairment.

Sixth, the EPAD study consists predominantly of mothers with depression and we were unable to consider whether or not findings extended to offspring of fathers with depression; patterns of co-occurring psychopathology are likely to differ by parent gender. Evidence has highlighted the importance of considering paternal depression as a risk for offspring psychopathology in its own right. ${ }^{35} \mathrm{~A}$ related point is that where one parent has depression, psychopathology in the co-parent may further increase risk to offspring. ${ }^{36}$ Alternatively, if the co-parent presents with no psychopathology this may act as a protective factor for offspring. ${ }^{37}$

Finally, many of the children in the current study developed psychiatric disorders other than depression; notably anxiety and disruptive behaviour disorders. It was not possible to delineate and compare risk for specific new-onset disorder types in this study. Further research should consider whether specific co-occurring problems in mothers with recurrent depression can help explain this heterogeneity in risk in offspring. Future research should also consider whether findings are the same or different for children of fathers with depression, the role of the co-parent, as well as address possible mechanisms involved in the link between parent and child psychopathology.

\section{Clinical implications}

Intervention studies suggest that effective treatment of parental depression can reduce child symptomotology. ${ }^{38}$ The current findings highlight the importance of clinicians also recognising additional psychopathology in mothers with depression, as their offspring are at especially high risk of developing psychopathology. These families should be prioritised for prevention and early intervention. $^{39}$

\footnotetext{
Ruth Sellers, BA, Stephan Collishaw, PhD, Institute of Psychological Medicine and Clinical Neurosciences, Cardiff University, MRC Centre for Neuropsychiatric Genetics and Genomics; Frances Rice, PhD, Department of Clinical, Educational and Health Psychology, University College London; Ajay K. Thapar MD, PhD, NISCHR, Institute of Psychological Medicine and Clinical Neurosciences, Cardiff University, MRC Centre for Neuropsychiatric Genetics and Genomics and Taff Riverside Practice, Cardiff; Robert Potter, MRCPsych, Institute of Psychological Medicine and Clinical Neurosciences, Cardiff University, MRC Centre for Neuropsychiatric Genetics and Genomics, Cardiff and Cwm Taf Health Board, Abercynon; Becky Mars, BSC, Institute of Psychological Medicine and Clinical Neurosciences, Cardiff University, MRC Centre for Medicine and Clinical Neurosciences, Cardiff University, MRC Centre for
Neuropsychiatric Genetics and Genomics, Cardiff; Gordon T. Harold, PhD, School of Psychology, College of Medicine, Biological Sciences and Psychology, University of Leicester; Daniel J. Smith, MD, MRCPysch, Institute of Health and Wellbeing, University of Glasgow; Michael J. Owen, FRCPsych, PhD, Institute of Psychological Medicine and Clinical Neurosciences, Cardiff University, MRC Centre for

Neuropsychiatric Genetics and Genomics, Cardiff; Nick Craddock, FRCPsych, PhD, Anita Thapar, FRCPsych, PhD, Institute of Psychological Medicine and Clinical Anita Thapar, FRCPsych, PhD, Institute of Psychological Medicine and Clinical
Neurosciences, Cardiff University, MRC Centre for Neuropsychiatric Genetics and Genomics, Cardiff, UK
}

Correspondence: Stephan Collishaw, Child \& Adolescent Psychiatry Section, Institute of Psychological Medicine and Clinical Neurosciences, School of Medicine, Cardiff University, 4th Floor B-C link Corridor, Heath Park, Cardiff CF14 4XN, UK. Email: collishaws@cf.ac.uk

First received 24 Oct 2011, final revision 23 July 2012, accepted 13 Aug 2012 


\section{Funding}

Sir Jules Thorn Charitable Trust, Waterloo Foundation.

\section{References}

1 Singh AL, D'Onofrio BM, Slutske WS, Turkheimer E, Emery RE, Harden KP et al. Parental depression and offspring psychopathology: a children of twins study. Psychol Med 2011; 41: 1385-95.

2 Weissman MM, Pilowsky DJ, Wickramaratne PJ, Talati A, Wisniewski SR, Fava $\mathrm{M}$, et al. Remission in maternal depression and child psychopathology: a STAR*D-child report. JAMA 2006; 296: 1389-98.

3 Avenevoli S, Merikangas KR. Implications of high-risk family studies for prevention of depression. Am J Prev Med 2006; 31 (6S1): S126-35.

4 Tully EC, lacono W, McGue M. An adoption study of parental depression and as environmental liability for adolescent depression and childhood disruptive disorders. Am J Psychiatry 2008; 165: 1148-54.

5 Silberg JL, Maes H, Eaves LJ. Genetic and environmental influences on the transmission of parental depression to children's depression and conduct disturbance: an extended children of twins study. J Child Psychol Psychiatry 2010; 51: 734-44.

6 Hammen C, Brennan PA. Severity, chronicity, and timing of maternal depression and risk for adolescent offspring diagnoses in a community samples. Arch Gen Psychiatry 2003; 60: 253-8.

7 Mars B, Collishaw S, Smith D, Thapar A, Potter R, Sellers R, et al. Offspring of parents with recurrent depression: which features of parent depression index risk for offspring psychopathology? J Affect Disord 2012; 136: 44-53.

8 Rohde P, Lewinsohn P, Seeley J. Comorbidity of unipolar depression: II. Comorbidity with other mental disorders in adolescents and adults. J Abnorm Psychol 1991; 100: 214-22.

9 Brown TA, Campbell LA, Lehman CL, Grisham JR, Mancill RB. Current and lifetime comorbidity of the DSM-IV anxiety and mood disorders in a large clinical sample. J Abnorm Psychol 2001; 110: 585-99.

10 Zoccolillo M. Co-occurrence of conduct disorder and its adult outcomes with depressive and anxiety disorders: a review. J Am Acad Child Adolesc Psychiatry 1992; 31: 547-56.

11 Grant BF, Harford TC. Comorbidity between DSM-IV alcohol use disorders and major depression: results of a national survey. Drug Alcohol Depend 1995; 39: 197-206.

12 Swendsen JD, Merikangas KR. The comorbidity of depression and substance use disorders. Clin Psychol Rev 2000; 20: 173-89.

13 Moffitt TE, Harrington $H$, Caspi A, Kim-Cohen J, Goldberg D, Gregory AM, et al. Depression and generalized anxiety disorder: cumulative and sequential comorbidity in a birth cohort followed prospectively to age 32 years. Arch Gen Psychiatry 2007; 64: 651-60.

14 Kessler R, DuPont R, Berglund $\mathrm{P}$, Wittchen $\mathrm{H}-\mathrm{U}$. Impairment in pure and comorbid generalized anxiety disorder and major depression at 12 months in two national surveys. Am J Psychiatry 1999; 156: 1915-23.

15 Kim-Cohen J, Moffitt TE, Taylor A, Pawlby SJ, Caspi A. Maternal depression and children's antisocial behavior: nature and nurture effects. Arch Gen Psychiatry 2005; 62: 173-81.

16 McLaughlin KA, Gadermann AM, Hwang I, Sampson NA, Al-Hamzawi A Andrade $\mathrm{LH}$, et al. Parent psychopathology and offspring mental disorders: results from the WHO World Mental Health Surveys. Br J Psychiatry 2012; 200: $290-9$.

17 American Psychiatric Association. Diagnostic and Statistical Manual of Mental Disorders (4th edn) (DSM-IV). APA, 1994.

18 Green H, McGinnity A, Meltzer H, Ford T, Goodman R. Mental Health of Children and Young People in Great Britain. TSO (The Stationery Office), 2004.

19 Beck AT, Rush AJ, Shaw BF, Emery G. Cognitive Therapy of Depression. Guilford Press, 1979
20 Wing JK, Barbor T, Brugha T, Burke J, Cooper JE, Giel R, et al. SCAN: Schedules for Clinical Assessment in Neuropsychiatry. Arch Gen Psychiatry 1990; 47: 589-93.

21 Zigmond AS, Snaith RP. The Hospital Anxiety and Depression Scale. Acta Psychiatr Scand 1983; 67: 361-70.

22 Achenbach TM, Rescorla LA. Manual for ASEBA Adult Forms and Profiles. University of Vermont, Research Center for Children, Youth, and Families, 2003.

23 Saunders JB, Aaland OG, Babor TF, De La Fuente JR, Grant M. Development of the Alcohol Use Disorders Identification Test (Audit): WHO collaborative project on early detection of persons with harmful alcohol consumption - II. Addiction 1993; 88: 791-803.

24 Angold A, Costello EJ. The Child and Adolescent Psychiatric Assessment (CAPA). J Am Acad Child Adolesc Psychiatry 2000; 39: 39-48.

25 Angold A, Costello E. A Test-retest reliability study of child-reported psychiatric symptoms and diagnoses using the Child and Adolescent Psychiatric Assessment (CAPA-C). Psychol Med 1995; 25: 755-62.

26 Royston P. Multiple imputation of missing values: further update of ice, with an emphasis on categorical variables. Stata J 2009; 9: 466-77.

27 Coryell W, Fiedorowicz JG, Solomon D, Leon AC, Rice JP, Keller MB. Effects of anxiety on the long-term course of depressive disorders. Br J Psychiatry 2012: 200: 210-5.

28 Kendler KS, Davis CG, Kessler RC. The familial aggregation of common psychiatric and substance use disorders in the National Comorbidity Survey: a family history study. Br J Psychiatry 1997; 170: 541-8.

29 O'Connor TG, McGuire S, Reiss D, Hetherington EM, Plomin R. Co-occurence of depressive symptoms and antisocial behaviour in adolescence: a common genetic liability. J Abnorm Psychol 1998; 107: 27-37.

30 Merikangas KR, Risch NJ, Weissman MM. Comorbidity and cotransmission of alcoholism, anxiety and depression. Psychol Med 1994; 24: 69-80.

31 Goodman SH, Gotlib IH. Risk for psychopathology in the children of depressed mothers: a developmental model for understanding mechanisms of transmission. Psychol Rev 1999; 106: 458-90.

32 Rice F, Harold GT, Shelton KH, Thapar A. Family conflict interacts with genetic liability in predicting childhood and adolescent depression. Am J Psychiatry 2006; 45: 841-8.

33 Carter AS, Garrity-Rokous FE, Chazan-Cohen R, Little C, Briggs-Gowan MJ. Maternal depression and comorbidity: predicting early parenting, attachment security, and toddler social-emotional problems and competencies. J Am Acad Child Adolesc Psychiatry 2001; 41: 18-26.

34 Kim-Cohen J, Caspi A, Rutter M, Tomas MP, Moffitt TE. The caregiving environments provided to children by depressed mothers with or without an antisocial history. Am J Psychiatry 2006; 163: 1009-18.

35 Ramchandani PG, Stein A, O'Connor TG, Heron J, Murray L, Evans J. Depression in men in the postnatal period and later child psychopathology: a population cohort study. J Am Acad Child Adolesc Psychiatry 2008; 47: 390-8.

36 Marmorstein NR, Malone SM, Iacono W. Psychiatric disorders among offspring of depressed mothers: associations with paternal psychopathology. Am J Psychiatry 2004; 161: 1588-94.

37 Brennan PA, Hammen C, Katz A, Le Brocque R. Maternal depression, paternal psychopathology, and adolescent diagnostic outcomes. J Consult Clin Psychol 2002; 70: 1075-85.

38 Pilowsky DJ, Wickramaratne $\mathrm{P}$, Talati A, Tang M, Hughes CW, Garber J, et al. Children of depressed mothers 1 year after the initiation of maternal treatment: findings from the STAR*D-Child Study. Am J Psychiatry 2008; 165: 1136-47.

39 Garber J, Clarke GN, Weersing VR, Beardslee WR, Brent DA, Gladstone TRG, et al. Prevention of depression in at-risk adolescents: a randomized controlled trial. JAMA 2009; 301: 2215-24. 